Bundesgesundheitsbl 2015 · 58:166-173 DOI 10.1007/s00103-014-2096-z

Online publiziert: 9. Dezember 2014

c) Springer-Verlag Berlin Heidelberg 2014
E. Fisher ${ }^{1} \cdot$ S. Achilles ${ }^{1} \cdot$ H. Tönnies ${ }^{1} \cdot$ J. Schmidtke ${ }^{2}$

${ }^{1}$ Geschäftsstelle der Gendiagnostik-Kommission, Robert Koch-Institut Berlin, Berlin, Deutschland

${ }^{2}$ Institut für Humangenetik, Medizinische Hochschule Hannover, Hannover, Deutschland

\title{
Konzepte zur Mitteilung genetischer Zusatzbefunde in der medizinischen Diagnostik und Forschung
}

Es kann wie ein Lotteriespiel sein: 2012 berichtet das TIME-Magazine vom Fall der 42-jährigen Englischlehrerin Laurie Hunter, die zur Klärung einer angeborenen Muskelschwäche ihrer 14-jährigen Tochter Amanda einer Genomsequenzierung des Kindes zustimmt. Die Untersuchung erbringt keinen Hinweis auf eine genetische Veränderung, die die Symptome ihrer Tochter erklären kann. Stattdessen stellen die Ärzte eine Deletion im Chromosom 1 fest, die für eine seltene Krebserkrankung verantwortlich sein kann. Dies konfrontiert die Mutter mit einem unvorhergesehenen, weiteren diagnostischen Klärungsbedarf: Da die Deletion auch bei ihr nachgewiesen wird, werden Mutter und Tochter einer Ganzkörper-MRT unterzogen. Lauries jüngstes Kind, die 2-jährige Tochter Kailyn, wurde mit einem Wolf-Hirschhorn-Syndrom geboren (dessen genetische Ursache jedoch nicht mit der Chromosomendeletion bei Laurie und Amanda in Verbindung steht). Nur der 4-jährige Sohn Ryan ist gesund. Nach langem Zögern entschließt sich die Mutter, Ryan auch auf die Deletion testen zu lassen, in Sorge darüber, er könne zu den seltenen Mutationsträgern zählen, bei denen der erste Tumor bereits im Kindesalter auftritt. Als sie erfährt, dass ihr Sohn nicht Träger des Chromosomendefekts ist, sei es gewesen „als könne ich wieder atmen“, so Laurie Hunter. Ihre eigene MRT zeigte eine Läsion oberhalb des Zwerchfells. Jetzt wartet sie darauf zu erfahren, ob es sich um einen Tumor handelt. Es sei oft einfacher, etwas nicht zu wissen, statt mit Informati- onen überwältigt zu werden, sagt sie und: "Now, it just feels like a waiting game" [1].

Seit etwa einem halben Jahrzehnt diskutieren Wissenschaftler, Ethiker, Juristen und Mediziner international den potenziellen Nutzen oder Schaden von genetischen Zusatzinformationen, die bei genomweiten Sequenziertechnologien als "Nebenprodukt" zum eigentlichen Untersuchungsziel anfallen können. Da sich die Anzahl der diesbezüglichen Begrifflichkeiten im Laufe der Zeit deutlich erhöht hat - im Allgemeinen war und ist vom "genetischen Zufallsbefund" (incidental genetic finding) die Rede - erscheint ein Überblick über die mittlerweile gebräuchlichen Bezeichnungen und Definitionen hilfreich. Ein genetischer Zufallsbefund ist nach allgemeiner Definition nicht intendiert (unsought [2,3] oder unsolicited [4]) und nicht erwartet (unanticipated) ${ }^{1}$. Die amerikanische Bioethikkommission ${ }^{2}$ hat eine weitere Differenzierung des $\mathrm{Zu}$ fallsbefundes vorgenommen, die sie anhand eines Beispiels erläutert: Bei einer molekulargenetischen Kompatibilitätstestung könne zufällig ein Vaterschaftsausschluss in einer untersuchten Familie festgestellt werden. Dabei handelt es sich zwar um einen nicht intendierten, aber vorhersehbaren und nicht vermeidbaren Befund (,An anticipatable incidental finding is a finding that is known to be

\footnotetext{
1 "Der Begriff „Zufallsbefund" [... ] bezeichnet einen unerwartet erhobenen Befund, für den zuvor keine erkennbaren Hinweise bestanden und der nicht im Rahmen einer gezielten Suche festgestellt wurde." [5].

2 Presidential Commission for the Study of Bioethical Issues (http://www.bioethics.gov).
}

\author{
Definition „Genetischer Zusatz- \\ befund" \\ Der genetische Zusatzbefund steht in kei- \\ nem Zusammenhang mit dem primären \\ Untersuchungsziel. Er ist nicht intendiert im \\ Sinne der eigentlichen medizinischen Frage- \\ stellung, die alleiniger Untersuchungszweck \\ ist, sondern ergibt sich aufgrund der ge- \\ zielten Auswertung zusätzlicher genetischer \\ Information.
}

associated with a test or procedure" [6]). Demgegenüber ist der unanticipatable incidental genetic finding ein Zufallsbefund, der nach gegenwärtigem Stand des Wissens nicht vorhersehbar ist. („This category includes a finding that could not ha$v e$ been anticipated given the current state of scientific knowledge" [6].) Anders verhält es sich beim genetischen Zusatzbefund (secondary finding oder secondary variant [7]). Dieser ergibt sich aufgrund eines aktiven Hinterfragens (etwa anhand einer zuvor erstellten Liste) von, für die untersuchte Person, relevanter genetischer Information, die jedoch nicht im Zusammenhang mit der ursprünglichen Fragestellung steht. Genetische Zusatzbefunde sind daher primär nicht intendiert, aber zu erwarten. Letzterer Terminus hat sich inzwischen als konzeptionell verbreitete Bezeichnung etabliert [7-9]. Für die proaktive Interpretation von genomischer Information im Hinblick auf genetische Zusatzbefunde wird die Bezeichnung des „opportunistischen Screenings" verwen$\operatorname{det}[10,11]$. Schließlich ist noch der Begriff der „genetischen Überschussinformation" zu nennen, die im definitorischen Sinne die gesamte, nicht zur Diagnosestellung benötigte und nicht als $\mathrm{Zu}$ - 
satzbefund in Frage stehende genetische Information bezeichnet [12].

Klinisch relevante (clinically actionable) genetische Zusatzbefunde heben sich gegenüber anderen Zufallsbefunden in der Medizin dadurch ab, dass sie zum einen oftmals prädiktiver Art sind dies unterscheidet sie z. B. von einem bei einer Röntgen- oder MRT-Diagnostik zufällig entdeckten Tumor - und zum anderen Implikationen für Angehörige oder Nachkommen der untersuchten Person haben können. Für die Aufklärung, deren Ziel es ist, die Patientin oder den $\mathrm{Pa}$ tienten in die Lage zu versetzen, eine informierte und selbstbestimmte Entscheidung darüber zu treffen, welche Ergebnisse einer genetischen Untersuchung sie oder er erfahren möchte, kann das Ausmaß der aufzuklärenden möglichen Zusatzbefunde bei einer vorgesehenen Exomsequenzierung (Whole Exome Sequencing, WES) oder Genomsequenzierung (Whole Genome Sequencing, WGS) Dimensionen erreichen, die sich bei den in der Diagnostik etablierten Untersuchungsmethoden wie der Array-CGH oder gezielten Sequenzierungen (PanelDiagnostik) nicht ergeben. Der Deutsche Ethikrat hob in seiner Stellungnahme zur genetischen Diagnostik die besonderen Herausforderungen genomweiter Analysen, insbesondere den Umgang mit nicht gewollten Zufallsbefunden, hervor: „Für den klinischen Einsatz besonders umfangreicher Gentests bis hin zur Exom- und Gesamtgenomsequenzierung sind Wege zu finden, [...] wie mit in diesem Kontext besonders zahlreich zu erwartenden Überschussinformationen und Nebenbefunden umzugehen ist. Dies gilt umso mehr, wenn umfangreiche Genomanalysen aufgrund weiterer technischer Fortschritte und Kostensenkungen künftig zu einer Standardmethode werden sollten " [13]. Aufgrund des zunehmenden Umfanges von Informationen und der gleichzeitig stärker "geforderten“ Patientenautonomie verweist der Deutsche Ethikrat auf eine notwendige Anpassung des Schutzkonzeptes für die betroffene oder ratsuchende Person und empfiehlt daher eine Ergänzung des Patientenschutzes um den Aspekt des Verbraucherschutzes. Einige seiner konkreten Forderungen beziehen sich auf das Gendiagnostikgesetz [14] und empfehlen u. a. eine gesetzliche Klarstellung im Hinblick auf die Art der Aufklärung bei der Nutzung der neuen diagnostischen Technologien (Empfehlung A7) sowie Anpassungen hinsichtlich der Aufklärung und der Untersuchung Minderjähriger (Empfehlung A8) [13]. Nach Auffassung der amerikanischen Bioethikkommission stellen für notwendige Anpassungen zum Schutz persönlicher Daten im Bereich der Genom- und Exomsequenzierungen untergesetzliche Regelungen eine geeignetere Alternative als Gesetzesneuerungen oder -ergänzungen dar. Untergesetzliche Regelungen können auf diesem Gebiet, das sich derzeit in einer raschen Entwicklung befindet, eher dem Stand der Wissenschaft entsprechende und in dem Sinne maßgeschneiderte Lösungsvorschläge liefern [15].

\section{Medizinisch bedeutsame genetische Datenbanken und technischer Stand von Genom- und Exomsequenzierungen}

In der Online Mendelian Inheritance in Men (OMIM) Datenbank ${ }^{3}$ sind aktuell ursächliche Genomveränderungen von 4174 monogenen Krankheiten gelistet, 1693 phänotypisch beschriebene Erbkrankheiten konnten bisher noch nicht molekulargenetisch charakterisiert werden [16]. Für 613 der einschließlich ihrer genetischen Ursache benannten Krankheiten gibt es in den GeneReviews detaillierte Informationen zur klinischen Diagnose, zu möglichen Behandlungsoptionen und spezifischen Aspekten der genetischen Beratung der betroffenen Personen und ihrer Familienangehörigen [17]. Das von der EU finanzierte Programm EuroGeneTest zur Harmonisierung genetischer Untersuchungen in den europäischen Staaten hat mittlerweile Leitlinien für 118 monogene Krankheiten veröffentlicht [18]. Darüber hinaus wurden seit 2005 in genomweiten Assoziationsstudien mehr als 10.000 mit spezifischen phänotypischen Eigenschaften (z. B. Körperfett, Größenwachstum, Lipidstatus etc.) sowie mit multifaktoriellen Erkrankungen assoziierte Genvarianten (sog. Single Nucleoti-

\footnotetext{
3 http://www.omim.org/statistics/entry (update am 20. August 2014).
}

de Variants oder kurz SNVs) beschrieben [19]. In der überwiegenden Zahl der Fälle kommt der einzelnen Variante jedoch keine klinische bzw. prognostische Relevanz zu. Durch Importieren von Gesamtgenomdaten in genomweite Assoziationsdatenbanken konnten inzwischen einige seltene SNVs (Häufigkeit < 1\%) identifiziert werden, deren prädiktive Aussagekraft sie unter Umständen als klinisch relevant klassifizieren kann (Bsp.: Eine seltene Variante des für den familiären Brustkrebs häufig verantwortlichen BRCA2Gens verdoppelt bei Rauchern das Lungenkrebsrisiko [20]).

Sequenzierungen des gesamten Exoms oder Genoms werden bisher nur selten in der klinischen Diagnostik eingesetzt. In den wenigen Fällen handelt es sich meist um schwere Erkrankungen des Neugeborenen- oder Kindesalters, für deren Diagnose andere Methoden versagt haben und die oftmals mit einer jahrelangen diagnostischen Odyssee der betroffenen Kinder und Familien verbunden waren. Im Bemühen, einen höheren und schnelleren Aufklärungserfolg dieser ausgesprochen seltenen Erkrankungen zu erreichen, wurden hohe Erwartungen in Exom- und Genomsequenzierungen gesetzt. Einige Tausend diagnostische Exomsequenzierungen sind in den letzten Jahren in den Vereinigten Staaten bereits durchgeführt worden [21]. Gesicherte Diagnosen konnten jedoch bisher nur in ca. $25 \%$ der Fälle gestellt werden [22]. Genomsequenzierungen, die auch nichtkodierende Bereiche mit einbeziehen und dabei potenziell pathogene Copy Number oder strukturelle Varianten ebenfalls erfassen, können bei einigen Erkrankungen Erfolg versprechender sein, wie eine kürzlich veröffentlichte Studie zeigte. In dieser konnte bei $42 \%$ der untersuchten Personen, die zuvor ergebnislos sowohl einer Panel-Diagnostik als auch einer Exomsequenzierung unterzogen worden waren, die genetische Ursache entdeckt werden [23]. Die Autoren schlossen aus den beobachteten Daten, dass in einem Patientenkollektiv, in dem eine Genomsequenzierung gleich zu Beginn durchgeführt würde, die Erfolgsrate bei $62 \%$ liegen könnte, wobei bei $60 \%$ der Betroffenen voraussichtlich eine (autosomal dominante oder 
$\mathrm{X}$-chromosomale) de-novo Mutation und bei $2 \%$ eine von beiden Elternteilen ererbte Krankheitsursache vorliegen könnte. Darüber hinaus werden in Zukunft internationale, frei zugängliche (Phänotyp-Genotyp-)Datenbanken (z. B. Exome Variant Server) oder Forschungsverbünde (z. B. International Rare Diseases Research Consortium) bei der Diagnosestellung seltener genetischer Defekte und der Suche nach Therapieansätzen für diese Erkrankungen hilfreich sein. Trotz der noch bestehenden Limitationen analytischer und interpretatorischer Art besteht die Vermutung, dass sich Genomsequenzierungen in der klinischen Praxis, nicht zuletzt aus ökonomischen Gründen, in Zukunft - auch als Instrument der Krankheitsvorsorge stärker durchsetzen könnten und möglicherweise irgendwann selbstverständlich in der Routinediagnostik oder im Neugeborenenscreening angewendet werden [24-26]. Gegenwärtig gilt der technische Standard (analytische Validität) von Genomsequenzierungen sowie die zweifelsfreie Annotation relevanter pathologischer Mutationen (klinische Validität) als nicht ausreichend für eine Zertifizierung als diagnostisches Verfahren [21, 27, 28]. Ende 2013 wurde zwar von der amerikanischen Zulassungsbehörde (US Food and Drug Administration, FDA) der erste Hochdurchsatzsequenzierautomat für die klinische Diagnostik lizensiert [29]. Noch ist dieser jedoch nicht für eine Gesamtgenomanalyse validiert. Die Gewinnung und Bereitstellung geeigneten Referenzmaterials aus kompletten menschlichen Genomen will die FDA gemeinsam mit dem National Institute for Standards and Technology bis Ende des Jahres abgeschlossen haben. Einsatz findet das Gerät bereits für die $\mathrm{Mu}$ tationsanalyse des CFTR ${ }^{4}$-Gens, dessen Veränderungen Mukoviszidose verursachen können ${ }^{5}$.

\footnotetext{
4 Cystic Fibrosis Transmembrane Conductance Regulator.

5 http://www.fda.gov/newsevents/newsroom/pressannouncements/ucm 375742.htm (Zugegriffen: 25.08.2014).
}

Bundesgesundheitsbl 2015 · 58:166-173 DOI 10.1007/s00103-014-2096-z

(c) Springer-Verlag Berlin Heidelberg 2014

E. Fisher · S. Achilles · H. Tönnies · J. Schmidtke

Konzepte zur Mitteilung genetischer Zusatzbefunde in der medizinischen Diagnostik und Forschung

\section{Zusammenfassung}

Die Hochdurchsatzsequenzierung gesamter Genome ist technisch bereits auf einem hohen Stand und wird als kostengünstigere Alternative zu anderen gezielten analytischen Verfahren in der klinischen Diagnostik genetischer Erkrankungen diskutiert. Andererseits besteht bei Genom- oder Exomsequenzierungen eine hohe Wahrscheinlichkeit, nicht mit dem primären Untersuchungsziel im Zusammenhang stehende Zusatzbefunde zu detektieren. Der vorliegende Beitrag versucht, einen Überblick über den ak- tuellen wissenschaftlichen und technischen Stand der Genom- und Exomsequenzierung sowie über die nationalen und internationalen Empfehlungen zum Umgang mit genetischen Zusatzbefunden, die vor allem im forschungsbezogenen und weniger im klinischen Kontext bereits zur Verfügung stehen, zu geben.

\section{Schlüsselwörter}

Genomsequenzierung - Exomsequenzierung . Zusatzbefunde $\cdot$ Mitteilung

\section{Concepts for the return of secondary genetic findings in medical diagnostics and research}

\begin{abstract}
High-throughput sequencing of whole genomes is technically already at a high level and is being discussed as a cost-effective alternative to other targeted, analytical procedures for clinical diagnosis of heritable disorders. On the other hand, with whole genome and whole exome sequencing, there is a high likelihood of uncovering secondary findings not associated with the primary aim of the investigation. This article tries to outline the current scientific and technical status of
\end{abstract}

whole genome and whole exome sequencing and of the national and international recommendations concerning the handling of secondary genetic findings which are already available, above all in the research-related context and less so in the clinical context.

\section{Keywords}

Whole genome sequencing - Whole exome sequencing $\cdot$ Secondary findings $\cdot$ Return of results

\section{Umgang mit genetischen Zusatzbefunden bei Genom- und Exomsequenzierungen im forschungsbezogenen Kontext}

Der Wunsch, möglichst umfassend genetische Variabilität aufDNA- und RNA-Ebene zu erfassen, um diese mit der Krankheitsentstehung korrelieren zu können, gab den Anstoß für bevölkerungsweite Genomsequenzierungsprojekte wie beispielsweise das 1000-Genome Project, das bereits kurz nach Vollendung des Humangenomprojekts 2003 begann [30] und in dem bis heute mehr als 2500 Genome sequenziert wurden ${ }^{6}$. Eine Reihe internationaler Forschungsinitiativen, zum Teil ko-

\footnotetext{
6 http://www.1000genomes.org/announcements/initial-phase-3-variant-list-and-phased-genotypes-2014-06-24 (Zugegriffen: 25.08.2014).
}

ordiniert vom Public Population Project in Genomics and Society, untersuchen mittlerweile Genome, Exome oder Transkriptome in großen Kollektiven. Diese Aktivitäten wiederum stellten den wesentlichen Anreiz für den rasanten technologischen Fortschritt der letzten Jahre auf dem Gebiet der Nukleinsäuresequenzierung dar: Kaum ein Jahrzehnt nach den noch sehr zeit- und kostenaufwendigen erstmaligen Sequenzierungen zweier individueller Genome $[31,32]$ sind heute Genomsequenzierungen im großen Maßstab technisch durchführbar.

Insbesondere in der Krebsforschung erhofft man sich durch den Aufbau umfangreicher Genomdatenbanken (die sowohl die Sequenzen der genetisch veränderten Tumorzellen als auch des konstitutionellen Genoms nicht betroffenen gesunden Gewebes enthalten), eine verbesserte differentielle Diagnostik und Prog- 


\section{Genomsequenzierung}

Die Rohsequenz eines menschlichen diploiden Genoms (6,6 Mrd. Basenpaare) kann innerhalb von 3 Werktagen analysiert werden, 2008 bedurfte es dafür noch 2 Monate [32]. Einschließlich der Qualitätskontrolle und Dateninterpretation kann eine Genomsequenzierung innerhalb von 1 Woche abgeschlossen werden [33]. Die Analysekosten (ausgenommen der Dateninterpretation und evtl. Reanalyse auffälliger Varianten) liegen derzeit unter US\$ $5000 .^{7}$

7 http://www.genome.gov/sequencingcosts/ (Zugegriffen: 25.08.2014); $1000 \$$ - Genom": http://wwwillumina.com/systems/hiseq-x-sequencing-system/system. ilmn (Zugegriffen: 25.08.2014).

nose von Krebserkrankungen, um maßgeschneiderte, personalisierte Behandlungsstrategien und Früherkennungsmöglichkeiten realisieren zu können. Für die an den Studien beteiligten Mediziner und Wissenschaftler stellte sich sehr früh die grundsätzliche Frage der Teilhabe der Studienteilnehmer an den persönlichen Untersuchungsergebnissen. Die in dem Zusammenhang sowohl aus wissenschaftlicher als auch ethischer und juristischer Perspektive diskutierten Fragen betrafen beispielsweise die Rückmeldepflicht der nichtärztlichen Wissenschaftler an die Studienärzte und die Durchführung der Aufklärung über Zusatzbefunde, die eine informierte Einwilligung der Studienteilnehmer in die Mitteilung derselben gewährleisten sollte. Im Marsilius-Kolleg der Universität Heidelberg hatte sich im März 2011 eine interdisziplinäre Arbeitsgruppe aus den Bereichen Medizin, Verfassungsrecht, Ethik, Naturwissenschaft, Informatik und Gesundheitsökonomie zusammengefunden, um zu diesen und anderen ethischen und rechtlichen Aspekten der Genomsequenzierung in der Forschung Lösungsvorschläge zu erarbeiten. Mit ihrer 2013 veröffentlichten Stellungnahme wurde in Deutschland erstmalig ein normativer Handlungskodex für die Aufklärung und die Einwilligung in die Mitteilung genetischer Zusatzbefunde bei Genomsequenzierungen erstellt [8]. Generell befürwortet die EURAT ${ }^{8}$-Grup-

8 „Ethische und Rechtliche Aspekte der Totalsequenzierung des menschlichen Genoms". pe die gezielte Auswertung und Mitteilung „medizinisch erheblicher" Zusatzbefunde 9 , wie „z. B. die genetischen Veränderungen, die erblichen Brustkrebs, erblichen Darmkrebs sowie bestimmte Herzmuskel- und Stoffwechselerkrankungen bedingen " und empfiehlt, diese im Rahmen der Aufklärung anhand von Beispielen zu erläutern. In der Aufklärung soll auch darüber informiert werden, welche Befundarten generell nicht ausgewertet und mitgeteilt werden. Dem Probanden soll die Möglichkeit gegeben werden, die Kenntnisnahme der Befunde abzulehnen (Opt-out)..$^{10} \mathrm{Ob}$ ein genetischer Zusatzbefund zwangsläufig mitgeteilt wird, ist auch abhängig von der Einschätzung des Arztes, der den Studienteilnehmer betreut. Ein Expertengremium soll künftig eine Leitlinie mittels eines empirischen Prozesses zur Spezifizierung von medizinisch erheblichen Zusatzbefunden entwickeln („Die Urteilsbildung über die Mitteilung von Zusatzbefunden soll in Heidelberg durch ein interdisziplinär besetztes Beratungsgremium und ein Erfahrungsregister unterstützt werden. Das Expertengremium, das sich in seiner Zusammensetzung an das EURAT-Gremium anlehnen könnte, dokumentiert und sammelt Entscheidungen über die Mitteilung von Zusatzbefunden. Es entwickelt Leitlinien und schreibt sie fort für die Entscheidung, welche Zusatzbefunde als medizinisch erheblich gelten sollen. Falls keine derartigen Leitlinien vorliegen, entscheidet der Arzt. "). In Großbritannien wird derzeit im Rahmen des von der britischen Regierung geplanten „100.000 Genome Projects" ein bevölkerungsweiter Survey ${ }^{11}$ durchgeführt, dessen Ergebnisse für die zukünftige Entwicklung einer Leitlinie zum Umgang mit nicht intendier-

\footnotetext{
9 Unter ",medizinisch erheblich“ fallen solche Zusatzbefunde, für die es A) zielgerichtete Therapie- und Vorsorgemaßnahmen gibt oder B) keine zielgerichtete Therapie- oder Vorsorgemaßnahmen gibt, die aber vielleicht wichtig für die Lebensplanung sind. (Musterformular "Patientenauskunft" der EURAT Stellungnahme [8]).

10 „Eine Rückmeldung von Zusatzbefunden sollte bereits in der Einwilligungserklärung und anhand einer adäquaten Aufklärung durch den Arzt - unter Beachtung des Rechts auf Nichtwissen - optional geregelt werden." [8].

11 http://www.genomicsengland.co.uk/ ethics-and-genomics-survey.html (Zugegriffen:
} 25.08.2014). ten genetischen Befunden genutzt werden soll. Zuvor hatte die Public Health Genomics Foundation in einem Grundsatzpapier zu dem geplanten Pilotprojekt klinischer Genomsequenzierungen Stellung bezogen [34]. Darin findet sich analog zum EURAT-Papier die Empfehlung, ausschließlich Ergebnisse mitzuteilen, die „clinically significant and severely or moderately life threatening and clinically actiona$b l e^{\text {“ sind. }}$

\section{Umgang mit Zusatzbefunden bei Genom- und Exomsequenzierungen im klinischen Kontext}

Ebenfalls 2013 hat nach 2-jähriger Beratungsphase eine Expertengruppe des American College of Medical Genetics and Genomics (ACMG) erstmalig Empfehlungen zum Umgang mit genetischen Zusatzbefunden in der klinischen Praxis veröffentlicht $[35,36]$. Diese enthalten eine Liste von 56 Genen assoziiert mit 24 Erkrankungen, für die es erwiesenermaßen wirksame präventive Maßnahmen oder Behandlungsmöglichkeiten gibt. Die Empfehlung lautet dahin gehend, dass die Genliste von dem mit der (Genom- oder Exom-) Sequenzierung beauftragten Labor prinzipiell ausgewertet werden muss und dabei gewonnene Informationen über unzweifelhaft pathogene Mutationen an den behandelnden Arzt weitergegeben werden. Die ACMG-Empfehlungen zielen im Wesentlichen darauf, ein potenzielles Over-Reporting genetischer Zusatzbefunde zu vermeiden. Die Schwelle für einen relevanten Mitteilungscharakter pathogener Mutationen müsse, so die Aussage der Verfasser der Empfehlungen, möglichst hoch angelegt werden: ,[...] it is imperative that a very high bar be set with return of only those variants with a very high probability of being deleterious" [36]. Nach ihrer vorläufigen Schätzung sollten lediglich $1 \%$ der untersuchten Personen Träger einer mitteilungsrelevanten Mutation sein. In einer neueren Studie, die 52 der in den Empfehlungen des ACMG benannten Gene bei 1000 Teilnehmern unterschiedlicher ethnischer Abstammung auswertete, zeigten sich als pathogen oder mit hoher Wahrscheinlichkeit pathogen eingestuf- 
te Zusatzbefunde bei 3,4 \% der Personen europäischer Abstammung [37]. Für die Befundmitteilung empfiehlt das ACMG der behandelnden ärztlichen Person ein oder mehrere Einzelgespräche mit dem Patienten zu führen, wobei dessen persönliche Situation, seine Krankheitsumstände und familiäre Situation angemessen berücksichtigt werden: „It is expected that the clinician will contextualize these findings to the clinical circumstances (e.g., the nature of ongoing clinical problems, knowledge of personal and family history, patient preferences, etc.) and the provider and patient will participate in a shared decision-making process regarding the return of results" [36]. Eine Opt-out-Möglichkeit der Auswertung und Weitergabe genetischer Zusatzbefunde des Labors an den behandelnden Arzt war in den Empfehlungen des ACMG von 2013 nicht vorgesehen. Sollte die betroffene Person nicht von medizinisch relevanten Zusatzbefunden Kenntnis nehmen wollen, hätte die Untersuchung nicht stattfinden können, es sei denn, der Arzt hätte nach persönlichem Ermessen entschieden, die Analyse dennoch in Auftrag zu geben. In diesem Fall hätte der Arzt sicherstellen und sorgfältig dokumentieren müssen, dass die Inanspruchnahme des Rechts auf Nichtwissen tatsächlich auf einer informierten Entscheidung beruht [38]. Darüber hinaus hätte er die betroffene Person darauf hinweisen müssen, dass der Untersuchungsbericht des Labors möglicherweise nicht aus der elektronischen Krankenakte entfernt werden kann [38]. Im Jahr 2014 hat das ACMG seine Empfehlungen nach Konsultation seiner Mitglieder jedoch revidiert und die Möglichkeit des Opt-outs bereits bei der Vornahme der genetischen Analyse eingeräumt [39]. Die Gesellschaft der Genetic Nurses and Counsellors in Großbritannien und Irland empfiehlt gleichfalls Gene, über die vor Untersuchungsbeginn aufgeklärt und anhand der Einwilligung eine Optout-Wahl des Patienten getroffen wurde, nicht auszuwerten, um zu vermeiden, dass das Labor oder der Arzt von genetischen Zusatzbefunden Kenntnis erhält, die die betroffene Person erklärtermaßen nicht wissen möchte [11]. Befürworter des Opt-outs sehen in einem konsequenten Vollzug des Rechts auf Nichtwissen einen wesentlichen Bestandteil des international anerkannten Patientenrechts ${ }^{12}$ [43], das jedem Menschen die Fähigkeit zugesteht, selbstverantwortlich über das eigene Wohl zu entscheiden [34, 43-45].

Die Befürworter einer proaktiven Kommunikation klinisch relevanter genetischer Zusatzbefunde berufen sich auf den ärztlichen Berufskodex, der die Fürsorge gegenüber dem Patienten als oberste Maxime ärztlichen Handelns ausweist. Die Deutsche Gesellschaft für Humangenetik (GfH) hält angesichts vermeidbarer Gesundheitsrisiken eine Non-disclosure-Strategie aus ärztlicher Sicht für ethisch nicht vertretbar: „Die Mitteilung von Zusatzbefunden, aus denen sich für die untersuchte Person ein relevantes Risiko für eine behandelbare Krankheit ergibt [...] ist nach Auffassung der GfH ärztlich geboten" [9]. Zu den Modalitäten eines Opt-outs hat sie sich nicht konkret geäußert. Deutlicher formuliert es die Europäische Gesellschaft für Humangenetik (ESHG) in ihren Empfehlungen zur Genomsequenzierung am Patienten, die auf eine, aus ärztlicher Sicht, im Einzelfall gebotene Beschränkung der Patientenautonomie hinweist: „Patient's claims to a right not to know do not automatically over-ride professional responsibilities when the patient's own health or that of his or her close relatives are at stake" (Recommendation no 5) [4].

Die Empfehlungen des ACMG beziehen sich uneingeschränkt auf alle Altersgruppen. Das bedeutet, auch bei der

12 UNESCO Universal Declaration on the Human Genome and Human Rights Article 5 c): "The right of each individual to decide whether or not to be informed of the results of genetic examination and the resulting consequences should be respected." [40]. Oviedo Convention on Human Rights and Biomedicine, Article 10 § 2: ,Everyone is entitled to know any information collected about his or her health. However, the wishes of individuals not to be so informed shall be observed." Article 10 § 3: In exceptional cases, restrictions may be placed by law on the exercise of the rights contained in paragraph 2 in the interests of the patient." [41]. Additional Protocol to the Convention on Human Rights and Biomedicine concerning Genetic Testing for Health Purposes, Chapter VII Article 16 § 2: Evveryone undergoing a genetic test is entitled to know any information collected about his or her health derived from this test" and Article $16 \S 3$ : "The wish of a person not to be informed shall be respected." [42].
Untersuchung von Kindern müssen alle Gene der Positivliste ausgewertet und etwaige Zusatzbefunde über behandelbare oder vermeidbare Erkrankungen des Erwachsenenalters in die Befundmitteilung eingeschlossen werden. Wenn anzunehmen ist, dass ein Elternteil ebenfalls von dem beim Kind festgestellten genetischen Erkrankungsrisiko betroffen sein kann, sei der Arzt - auch im Sinne des Kindeswohls zur Mitteilung an die Eltern verpflichtet [36]. Die Gesellschaft der Genetic Nurses and Counsellors hat sich deutlich von den ACMG-Empfehlungen distanziert und fordert, ein opportunistisches Screening bei Kindern nicht routinemäßig auf Erkrankungen des Erwachsenenalters auszudehnen [11]. In der GfH-Stellungnahme heißt es dazu: „Falls sich aus dem bei einer nicht-einwilligungsfähigen Person oder bei Ungeborenen erhobenen Zusatzbefund direkte medizinische Konsequenzen für Angehörige ergeben, ist im Sinne der Wahrung der Anliegen Dritter (sog. Drittschutz) eine ärztliche Abwägung erforderlich, ob dieses Ergebnis den gesetzlichen Vertretern mitzuteilen ist. "Der Arzt müsse „im Einzelfall den Schutz von NichtEinwilligungsfähigen gegenüber dem Recht auf Wissen von Verwandten werten und gewichten" [9].

Die vom ACMG erstellte Positivliste von Erkrankungen bzw. Genen, die als medizinisch relevant eingestuft werden, blieb in Folge keineswegs unkritisch kommentiert [45, 46]. Als Kriterien für die medizinische Relevanz - den klinischen Nutzen und die klinische Validität benannte das ACMG die bekannte oder erwartete („with a very high probability of being deleterious “ [36]) Pathogenizität einer genetischen Variante, die Behandelbarkeit oder Präventionsmöglichkeit der assoziierten Erkrankung und, sofern vorhanden, die Möglichkeit einer diagnostischen Konfirmation. Ein großes Problem stellt die Zuordnung neuer Varianten der benannten Gene in eine der drei Kategorien „gesichert pathogen“, „höchstwahrscheinlich pathogen“ oder „unbedenklich“ dar [46]. Nicht selten münden Beurteilungsversuche genetischer Varianten weder in ein definitiv positiv noch negativ gesichertes Ergebnis. Dies gilt sowohl für die indikationsspezifischen als auch nicht intendierten Befunde. Sie werden als $\mathrm{Zu}$ - 


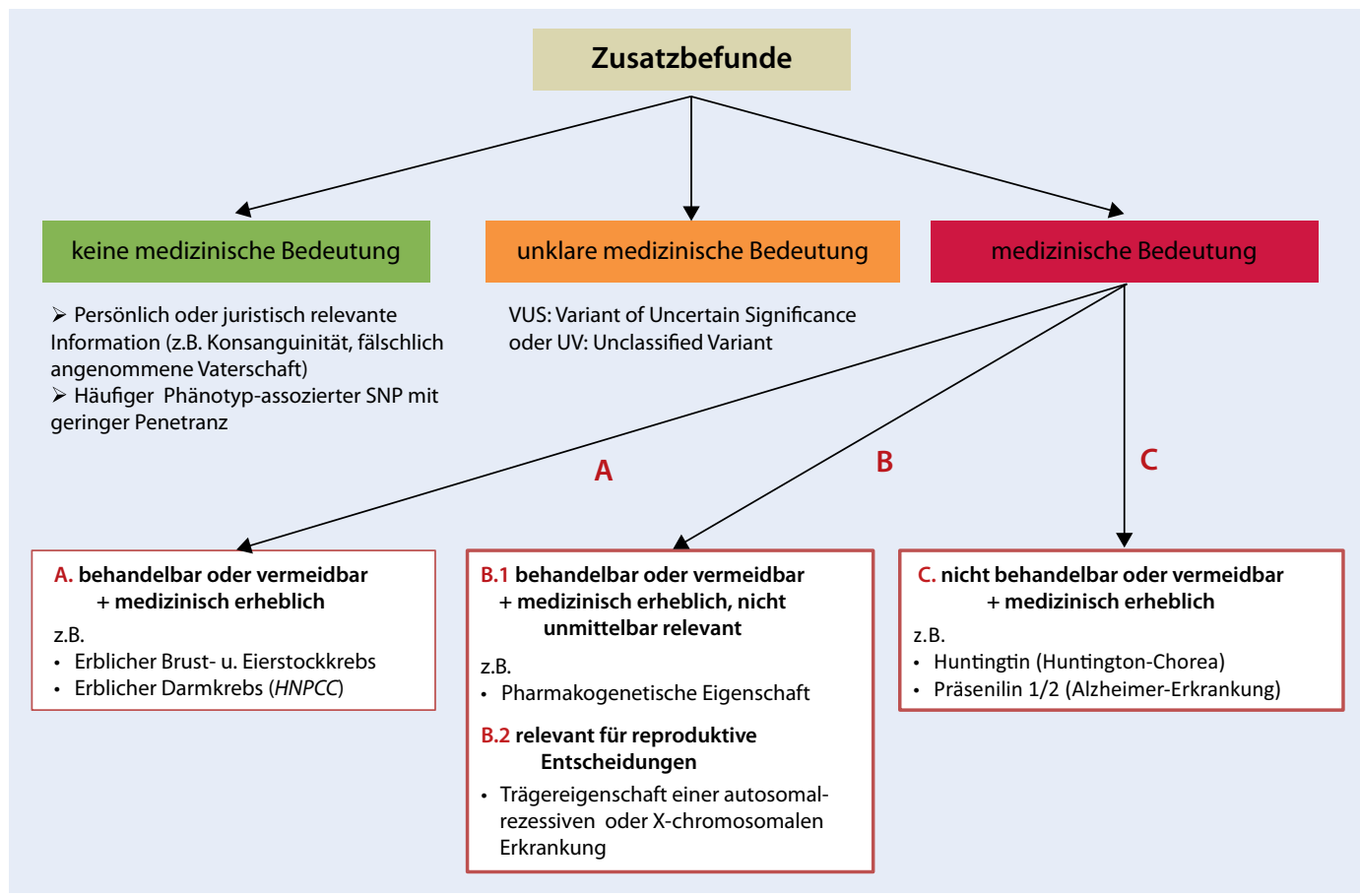

Abb. $1 \varangle$ Kategorisierung genetischer Zusatzbefunde. Die Abbildung ist stark verallgemeinernd und soll wertefrei im Hinblick auf die Mitteilungsrelevanz der einzelnen Gruppen sowohl im klinischen als auch forschungsbezogenen Kontext sein. Die Kennzeichnung $A-C$ soll die, in dem Sinne "wertungslose" Aufzählung klinisch relevanter genetischer Eigenschaften verdeutlichen. Prinzipiell ist für alle in der Abbildung bezeichneten Gruppen im Einzelnen zu beurteilen, ob diese als Zusatzbefunde ausgewertet und sofort mitgeteilt werden, in die elektronische Krankenakte aufgenommen oder ignoriert bzw. nicht ausgewertet oder durch Filter ausgeschlossen werden sollten

satzbefunde mit unklarer Bedeutung ( $v a$ riants of uncertain significance, VUS oder auch unclassified variants, UVs) im Befundbericht des Labors dokumentiert und kommuniziert. Die ESHG schreibt in ihren Empfehlungen: „It is important that findings of uncertain significance are included in reports, as their significance may become clear at a later date" [47]. Welche Handlungsoption aber soll der übermittelnden ärztlichen Person in Bezug auf diese unklaren Befunde gegeben werden? VUS stellen aus ethischer Sicht eine besondere Herausforderung dar. Es kann der Fall eintreten, dass ein derartiger Befund nicht an die untersuchte Person weitergegeben wurde, in der Folgezeit aber als eindeutig pathogen eingestuft werden kann und zu diesem Zeitpunkt der betroffenen Person keine Möglichkeit der Kenntnisnahme dieser Information gegeben ist. Andererseits können durch die Mitteilung nicht validierter Befunde auch unnötige Ängste entstehen und überflüssige Untersuchungen in Folge stattfinden. Im Hinblick auf diese Befunde stellt sich daher die Frage, inwieweit eine Rekontaktierung der untersuchten Person möglich sein sollte. Im Rahmen von zeitlich begrenzten Forschungsvorhaben ist diese Möglichkeit im Allgemeinen auf die Projektlaufzeit beschränkt [48]. Im klinischen Kontext sind rechtli- che Ansprüche oder die Frage, über welchen Zeitraum ein erneuter Kontakt zum Patienten stattfinden könnte, derzeit noch unklar. Die ESHG hat hierfür eine klare Leitlinie gefordert [4].

\section{Klassifizierung von Zufallsbefunden}

Erstmalig stellten Berg et al. 2011 eine Unterteilung genetischer Zufallsbefunde nach Relevanz und klinischem Nutzen vor [49]. Die Autoren bildeten drei Kategorien (sog. bins). In die erste Kategorie fallen genetische Eigenschaften, deren Nachweis unmittelbare Konsequenzen für die Prävention und Behandelbarkeit einer Erkrankung haben, z. B. Mutationen, die für hereditären Darmkrebs (Lynch-Syndrom) prädisponieren. In die zweite Kategorie fallen genetische Varianten, die wissenschaftlich validiert, jedoch zum Untersuchungszeitpunkt noch keine klinische Relevanz haben, z. B. pharmakogenetische Eigenschaften, die erst bei einer Anwendung des Medikaments von Bedeutung sind, oder Trägereigenschaften für rezessive Erkrankungen. Zu dieser Kategorie zählen die Autoren auch genetische Eigenschaften, die für nicht behandelbare oder vermeidbare Erkrankungen (z. B. Huntington-Chorea) verantwortlich oder mitverantwortlich sind (z. B. ApoE-4 als Risikofaktor für die Alzheimer-Erkrankung). Der dritten Kategorie ordnen Berg et al. schließlich alle anderen genetischen Eigenschaften, deren Aussagekraft für eine gesundheitliche Beeinträchtigung wissenschaftlich (noch) nicht validiert ist, zu. Letztere Kategorie sei, so die Autoren, im klinischen Untersuchungskontext typischerweise keine mitteilungsrelevante Gruppe, jedoch für die zukünftige Forschung von Bedeutung. Es gibt darüber hinaus inzwischen weitere, zum Teil detailliertere Modelle der Klassifizierung genetischer Zufallsbefunde in der Literatur, z. B. [34, 50, 51], auf die hier jedoch nicht näher eingegangen wird. Im Wesentlichen finden sie ihre Übereinstimmung in der Abgrenzung dreier klinisch relevanter Gruppen von Zusatzbefunden: a) genetische Eigenschaften für unmittelbar medizinisch erhebliche und behandelbare oder vermeidbare Erkrankungen, b) nicht unmittelbar medizinisch erhebliche genetische Eigenschaften oder Trägereigenschaften für autosomal-rezessive oder X-chromosomale Erkrankungen und c) genetische Eigenschaften für medizinisch erhebliche, jedoch nicht behandelbare oder vermeidbare Erkrankungen (- Abb. 1).

Zusammenfassend lässt sich feststellen, dass sich trotz rechtlicher und ethischer Unterschiede bei der Frage des Um- 
gangs mit genetischen Zusatzbefunden in der klinischen Praxis und im Forschungskontext die im Rahmen von wissenschaftlichen Forschungsprojekten vorgeschlagenen und teilweise schon umgesetzten Verfahrensweisen möglicherweise in analoger Weise in der klinischen Praxis bewähren können. Dies betrifft insbesondere die Aufklärung, Einwilligung und die Berücksichtigung des Rechts auf Nichtwissen des Patienten.

\section{Korrespondenzadresse}

\section{Dr. E. Fisher}

Geschäftsstelle der Gendiagnostik-Kommission Robert Koch-Institut Berlin

Nordufer 20, 13353 Berlin

fishere@rki.de

\section{Einhaltung ethischer Richtlinien}

Interessenkonflikt. E. Fisher, S. Achilles, H. Tönnies und J. Schmidtke geben an, dass kein Interessenkonflikt besteht.

\section{Literatur}

1. Rochman B (2012) The DNA dilemma: a test that could change your life. TIME Magazine 180:30-35

2. Health Council of the Netherlands (2010) The ,thousand-dollar genome': an ethical exploration. - The Hague: centre of ethics and health. Monitoring report ethics and health 2010/2012. http:// www.gezondheidsraad.nl/sites/default/files/ 201015E.pdf. Zugegriffen: 25. Aug. 2014

3. Metaforum Leuven (2011) Full sequencing of the human genome. Metaforum paper 6 - Katholieke Universiteit Leuven. https://www.kuleuven.be/ metaforum/docs/pdf/wg_14_e.pdf. Zugegriffen: 25. Aug. 2014

4. European Society of Human Genetics (2013) Whole-genome sequencing in health care. Recommendations of the European Society of Human Genetics. Eur J Hum Genet 21:580-584

5. Deutscher Bundestag (2009) Drucksache 16/12000: Zukunftsreport: Individualisierte Medizin und Gesundheitssystem. (Drs.16/12000). http://dipbt.bundestag.de/dip21/ btd/16/120/1612000.pdf. Zugegriffen: 25. Aug. 2014

6. Presidential Commission for the Study of Bioethical Issues (2013) Anticipate and communicate: ethical management of incidental and secondary findings in the clinical, research, and direct-to-consumer contexts. - Washington D.C. http://bioethics.gov/sites/default/files/FINALAnticipateCommunicate_PCSBI_0.pdf.ZZugegriffen: 25. Aug. 2014

7. Christenhusz GM, Devriendt K, Dierickx K (2013) Secondary variants - in defense of a more fitting term in the incidental findings debate. Eur J Hum Genet 21:1331-1334
8. Ethische und rechtliche Aspekte der Totalsequenzierung (EURAT) Projekt-Gruppe (2013) Eckpunkte für eine Heidelberger Praxis der Ganzgenomsequenzierung. http://www.marsilius-kolleg.uniheidelberg.de/md/einrichtungen/mk/presse/stellungnahme_heidelberger_praxis_der_ganzgenomsequenzierungl_2013-06-12.pdf.ZZugegriffen: 25. Aug. 2014

9. Deutsche Gesellschaft für Humangenetik (2013) Stellungnahme zu genetischen Zusatzbefunden in Diagnostik und Forschung. Med Genet 25(2):184186. http://www.gfhev.de/de/leitlinien/LL_und_ Stellungnahmen/2013_05_28_Stellungnahme_ zu_genetischen_Zufallsbefunden.pdf. Zugegriffen: 25. Aug. 2014

10. Burke W, Matheny Antommaria AH, Bennett R et al (2013) Recommendations for returning genomic incidental findings? We need to talk! Genet Med 15:854-859

11. Association of Genetic Nurses and Counsellors (UK and Ireland) (2014) Position statement on opportunistic genomic screening from the Association of Genetic Nurses and Counsellors (UK and Ireland). Eur J Hum Genet 22(8):955-956

12. Nationale Akademie der Wissenschaften, Deutsche Akademie der Technikwissenschaften, BerlinBrandenburgische Akademie der Wissenschaften (2010) Prädiktive genetische Diagnostik als Instrument der Krankheitsprävention. http://www.leopoldina.org/uploads/tx_leopublication/201011_ natEmpf_praedikative-DE.pdf. Zugegriffen: 25. Aug. 2014

13. Deutscher Ethikrat (2013) Die Zukunft der genetischen Diagnostik - von der Forschung in die klinische Anwendung. http://www.ethikrat.org/dateien/pdf/stellungnahme-zukunft-der-genetischendiagnostik.pdf. Zugegriffen: 28. Aug. 2014

14. Bundesministerium für Gesundheit (2009) Gesetz über genetische Untersuchungen bei Menschen (Gendiagnostikgesetz - GenDG). BGBI Teil I (50):2529-2538. http://www.gesetze-im-internet. de/bundesrecht/gendg/gesamt.pdf. Zugegriffen: 25. Aug. 2014

15. Presidential Commission for the Study of Bioethical Issues (2012) Privacy and progress in whole genome sequencing. - Washington D.C. http://www. bioethics.gov/cms/sites/default/files/PrivacyProgress508.pdf. Zugegriffen: 25. Aug. 2014

16. Online Mendelian Inheritance in Man (OMIM) (2013) McKusick-Nathans Institute of Genetic Medicine, Johns Hopkins University (Baltimore, MD). (letzter Update: 20. Aug. 2014). http://www.ncbi. nlm.nih.gov/Omim/mimstats.html. Zugegriffen: 25. Aug. 2014

17. GeneReviews [Internet] Seattle (WA) (ohne Datum) University of Washington, Seattle. http:// www.ncbi.nlm.nih.gov/books/NBK1116/.Zugegriffen: 25. Aug. 2014

18. EuroGentest (2013) Clinical utility gene cards. (letzter Update: 23. July 2014). http://www.eurogentest.org/index.php?id=668. Zugegriffen: 25 . Aug. 2014

19. Hindorff LA, MacArthur J, Morales J et al (2014) A catalog of published genome-wide association studies. (letzter Update: 8. Aug. 2014). www.genome.gov/gwastudies. Zugegriffen: 25. Aug. 2014

20. Wang Y, McKay JD, Rafnar T et al (2014) Rare variants of large effect in BRCA2 and CHEK2 affect risk of lung cancer. Nat Genet 46:736-741

21. Biesecker LG, Green RC (2014) Diagnostic clinical genome and exome sequencing. N Engl J Med 370:2418-2425
22. Yang Y, Muzny DM, Reid JG et al (2013) Clinical whole-exome sequencing for the diagnosis of mendelian disorders. N Engl J Med 369:1502-1511

23. Gilissen C, Hehir-Kwa JY, Thung DT et al (2014) Genome sequencing identifies major causes of severe intellectual disability. Nature 511:344-347

24. Dondorp WJ, de Wert GM, Niermeijer MF (2012) Genomic sequencing in newborn screening programs. JAMA 307:2146. (author reply)

25. Greeley SA, Msall ME, Acharya K (2012) Genomic sequencing in newborn screening programs. JAMA 307:2146-2147. (author reply)

26. Knoppers BM, Senecal K, Borry P, Avard D (2014) Whole-genome sequencing in newborn screening programs. Sci Transl Med 6:229cm222

27. PHG Foundation (2014) Clinical whole genome analysis: delivering the right diagnosis. http:// www.phgfoundation.org/file/15238/. Zugegriffen: 25. Aug. 2014

28. Feero W (2014) Clinical application of whole-genome sequencing: proceed with care. JAMA 311:1017-1019

29. Collins FS, Hamburg MA (2013) First FDA authorization for next-generation sequencer. $\mathrm{N}$ Engl J Med 369:2369-2371

30. Kaiser J (2008) A plan to capture human diversity in 1000 genomes. Science 319:395

31. Levy S, Sutton G, Ng PC et al (2007) The diploid genome sequence of an individual human. PLoS Biol 5:e254. http://www.plosbiology.org/article/ info\%3Adoi\%2F10.1371\%2Fjournal.pbio.0050254. Zugegriffen: 25. Aug. 2014

32. Wheeler DA, Srinivasan M, Egholm M et al (2008) The complete genome of an individual by massively parallel DNA sequencing. Nature 452:872-876

33. Johansen Taber KA, Dickinson BD, Wilson M (2014) The promise and challenges of next-generation genome sequencing for clinical care. JAMA Intern Med 174:275-280

34. PHG Foundation (2013) Managing incidental and pertinent findings from WGS in the $100,000 \mathrm{Ge}$ nome Project - A discussion paper from the PHG Foundation. http://www.phgfoundation.org/ news/13721/. Zugegriffen: 25. Aug. 2014

35. American College of Medical Genetics (2013) ACMG recommendations for reporting of incidental findings in clinical exome and genome sequencing. Genet Med 15(7):565-574. https://www. acmg.net/docs/IF_Statement_Final_7.24.13.pdf. Zugegriffen: 25. Aug. 2014

36. American College of Medical Genetics (2013) Incidental findings in clinical genomics: a clarification: American College of Medical Genetics and Genomics. Genet Med 15(8):664-666. https://www. acmg.net/docs/Incidental_Findings_in_Clinical_ Genomics_A_Clarification_081413.pdf. Zugegriffen: 25. Aug. 2014

37. Dorschner MO, Amendola LM, Turner EH et al (2013) Actionable, pathogenic incidental findings in 1,000 participants' exomes. Am J Hum Genet 93:631-640

38. McGuire AL, Joffe S, Koenig BA et al (2013) Ethics and genomic incidental findings. Science 340:1047-1048

39. American College of Medical Genetics (2014) ACMG updates recommendation on "Opt Out" for genome sequencing return of results. https:// www.acmg.net/docs/Release_ACMGUpdatesRecommendations_final.pdf. Zugegriffen: 01. Aug. 2014 
40. UNESCO (1997) Universal Declaration on the Human Genome and Human Rights (11 November 1997) In: UNESCO (Hrsg) Resolutions. - Paris: Unesco. - (Records of the General Conference: Twenty-ninth Session/ Paris/ 21 October to 12 November 1997; Bd 1 (Chapter III 16)). S 41-46. http://www.unesco.org/new/en/social-and-human-sciences/themes/bioethics/human-genomeand-human-rights/. Zugegriffen: 25. Aug. 2014

41. Council of Europe (1997) Convention for the protection of human rights and dignity of the human being with regard to the application of biology and medicine: convention on human rights and biomedicine. http://conventions.coe.int/Treaty/en/Treaties/Word/164.doc. Zugegriffen: 25. Juli 2011

42. Council of Europe (2008) Additional protocol to the convention on human rights and biomedicine, concerning genetic testing for health purposes. http://conventions.coe.int/Treaty/EN/Treaties/ Html/203.htm. Zugegriffen: 05. Nov. 2010

43. Doppelfeld E (2013) Communication of results and incidental findings in medical research - a european perspective: World Medical Association, Inc. World Med J 59(6). http://www.wma.net/ en/30publications/20journal/pdf/wmj201306.pdf. Zugegriffen: 25. Aug. 2014

44. Townsend A, Rousseau F, Friedman J, Adam S, Lohn Z, Birch P (2014) Autonomy and the patient's right,not to know' in clinical whole-genomic sequencing. Eur J Hum Genet 22:6-11

45. Wolf SM, Annas GJ, Elias S (2013) Patient autonomy and incidental findings in clinical genomics. Science 340:1049-1050

46. Holtzman NA (2013) ACMG recommendations on incidental findings are flawed scientifically and ethically. Genet Med 15:750-751

47. European Society of Human Genetics (2014) Recommendations for reporting results of diagnostic genetic testing (biochemical, cytogenetic and molecular genetic). Eur J Hum Genet 22(2):160-170. http://www.nature.com/ejhg/journal/v22/n2/pdf/ ejhg2013125a.pdf. Zugegriffen: 25. Aug. 2014

48. Fabsitz RR, McGuire A, Sharp RR et al (2010) Ethical and practical guidelines for reporting genetic research results to study participants: updated guidelines from a National Heart, Lung, and Blood Institute working group. Circ Cardiovasc Genet 3(6):574-580. http://www.ncbi.nlm.nih.gov/pmc/ articles/PMC3090664/pdf/nihms272954.pdf. Zugegriffen: 25. Aug. 2014

49. Berg JS, Khoury MJ, Evans JP (2011) Deploying whole genome sequencing in clinical practice and public health: meeting the challenge one bin at a time. Genet Med 13:499-504

50. Ayuso C, Millan JM, Mancheno M, Dal-Re R (2013) Informed consent for whole-genome sequencing studies in the clinical setting. Proposed recommendations on essential content and process. Eur J Hum Genet 11:1054-1059

51. Rudnik-Schöneborn $S$, Langanke $M$, Erdmann $P$, Robienski J (2014) Ethische und rechtliche Aspekte im Umgang mit genetischen Zufallsbefunden - Herausforderungen und Lösungsansätze. Ethik Med 26:105-119 